\title{
Mapping far-IR emission from the central kiloparsec of NGC $1097^{\star}$
}

\author{
K. Sandstrom ${ }^{1}$, O. Krause ${ }^{1}$, H. Linz ${ }^{1}$, E. Schinnerer ${ }^{1}$, G. Dumas ${ }^{1}$, S. Meidt ${ }^{1}$, H.-W. Rix ${ }^{1}$, M. Sauvage ${ }^{2}$, F. Walter ${ }^{1}$, \\ R. C. Kennicutt ${ }^{3}$, D. Calzetti ${ }^{4}$, P. Appleton ${ }^{5}$, L. Armus ${ }^{6}$, P. Beirão ${ }^{6}$, A. Bolatto ${ }^{7}$, B. Brandl ${ }^{8}$, A. Crocker ${ }^{4}$, K. Croxall ${ }^{9}$, \\ D. Dale ${ }^{10}$, B. T. Draine ${ }^{11}$, C. Engelbracht ${ }^{12}$, A. Gil de Paz ${ }^{13}$, K. Gordon ${ }^{14}$, B. Groves ${ }^{8}$, C.-N. Hao ${ }^{15}$, G. Helou ${ }^{5}$, \\ J. Hinz ${ }^{12}$, L. Hunt ${ }^{16}$, B. D. Johnson ${ }^{3}$, J. Koda ${ }^{17}$, A. Leroy ${ }^{18}$, E. J. Murphy ${ }^{6}$, N. Rahman ${ }^{7}$, H. Roussel ${ }^{19}$, R. Skibba ${ }^{12}$, \\ J.-D. Smith ${ }^{9}$, S. Srinivasan ${ }^{19}$, L. Vigroux ${ }^{19}$, B. E. Warren ${ }^{20}$, C. D. Wilson ${ }^{21}$, M. Wolfire ${ }^{7}$, and S. Zibetti ${ }^{1}$
}

(Affiliations are available in the online edition)

Received 30 March 2010 / Accepted 29 April 2010

\begin{abstract}
Using photometry of NGC 1097 from the Herschel PACS (Photodetector Array Camera and Spectrometer) instrument, we study the resolved properties of thermal dust continuum emission from a circumnuclear starburst ring with a radius $\sim 900 \mathrm{pc}$. These observations are the first to resolve the structure of a circumnuclear ring at wavelengths that probe the peak (i.e. $\lambda \sim 100 \mu \mathrm{m}$ ) of the dust spectral energy distribution. The ring dominates the far-infrared (far-IR) emission from the galaxy - the high angular resolution of PACS allows us to isolate the ring's contribution and we find it is responsible for 75, 60 and 55\% of the total flux of NGC 1097 at 70, 100 and $160 \mu$ m, respectively. We compare the far-IR structure of the ring to what is seen at other wavelengths and identify a sequence of far-IR bright knots that correspond to those seen in radio and mid-IR images. The mid- and far-IR band ratios in the ring vary by less than $\pm 20 \%$ azimuthally, indicating modest variation in the radiation field heating the dust on $\sim 600 \mathrm{pc}$ scales. We explore various explanations for the azimuthal uniformity in the far-IR colors of the ring including a lack of well-defined age gradients in the young stellar cluster population, a dominant contribution to the far-IR emission from dust heated by older (>10 Myr) stars and/or a quick smoothing of local enhancements in dust temperature due to the short orbital period of the ring. Finally, we improve previous limits on the far-IR flux from the inner $\sim 600 \mathrm{pc}$ of NGC 1097 by an order of magnitude, providing a better estimate of the total bolometric emission arising from the active galactic nucleus and its associated central starburst.
\end{abstract}

Key words. infrared: galaxies - galaxies: star formation - galaxies: individual: NGC 1097 - dust, extinction

\section{Introduction}

The central regions of galaxies host some of the most intense star-formation that we can observe in the local Universe in circumnuclear starburst rings. Starburst rings are believed to be the consequence of the pile-up of inflowing gas and dust, driven by a non-axisymmetric potential from a stellar bar, on orbits located near the inner Lindblad resonance of the bar (Combes \& Gerin 1985; Athanassoula 1992). The high surface densities that exist in the ring lead to high star-formation rates. Indeed starburst rings are one of few regions in non-interacting galaxies where the formation of "super star clusters" commonly occurs (Maoz et al. 1996). The stars formed in the ring can be numerous enough to drive the structural evolution of the galaxy (Norman et al. 1996; Kormendy \& Kennicutt 2004) and can be the dominant power source for the galaxy's infrared (IR) emission.

Star-formation in circumnuclear rings occurs under conditions not normally found in the disks of galaxies: in addition to their high gas surface densities, these regions have dynamical timescales that are comparable to the lifetimes of massive stars. Understanding star formation in circumnuclear rings has been a long-standing problem (Combes 1996). There are two

* Herschel is an ESA space observatory with science instruments provided by European-led Principal Investigator consortia and with important participation from NASA. main models: the "popcorn" model (Elmegreen 1994), where star-formation is driven by stochastic gravitational fragmentation along the ring, and the "pearls on a string" model, where gas flowing into the ring is compressed near the contact points (i.e. locations where the dust lanes intersect the ring) and then forms stars a short distance downstream (e.g., Böker et al. 2008). The "pearls on a string" model predicts a gradient in the ages of young stellar clusters as one moves away from the contact points. This has been observed in a number of starburst rings (e.g., Mazzuca et al. 2008; Böker et al. 2008). Conversely, many well-studied rings show no evidence for an age gradient (Maoz et al. 2001). It is not obvious, however, that a single mode of star-formation must occur in all rings or even at all times in a given ring (van de Ven \& Chang 2009).

KINGFISH (Key Insights on Nearby Galaxies: a FarInfrared Survey with Herschel, PI R. Kennicutt) is an opentime key program to study the interstellar medium (ISM) of nearby galaxies with far-IR/sub-mm photometry and spectroscopy. Among the unique aspects of the KINGFISH science program is the ability to observe thermal dust emission at unprecedented spatial resolution ( 5.6, 6.8 and $11.3^{\prime \prime}$ at 70, 100 and $160 \mu \mathrm{m}$ ) using PACS (Photodetector Array Camera and Spectrometer) imaging. High spatial resolution is crucial for observing processes occurring in the central regions of galaxies. These regions represent our best opportunity to study in detail 
the interplay between dynamics, star-formation and feedback that regulate the fueling of nuclear activity, be it a starburst or an active galactic nucleus (AGN).

Below we present PACS imaging of the galaxy NGC 1097, one of the first KINGFISH targets observed during the Herschel science demonstration program (SDP) (for PACS spectroscopy of NGC 1097 see Beirão et al. 2010; and for SPIRE observations see Engelbracht et al. 2010). The source NGC 1097 is a barred spiral galaxy located at a distance of $19.1 \mathrm{Mpc}$ (Willick et al. $\left.1997,1^{\prime \prime} \approx 92 \mathrm{pc}\right)$. In its central kpc it hosts an intensely starforming $\left(\sim 5 M_{\odot} \mathrm{yr}^{-1}\right.$; Hummel et al. 1987) ring with a radius of $\sim 900 \mathrm{pc}$. The ring's rotation speed of $\sim 300 \mathrm{~km} \mathrm{~s}^{-1}$ (corrected for inclination, Storchi-Bergmann et al. 1996), corresponds to a rotation period of $\sim 18$ Myr. The galaxy's nucleus is classified as a LINER from optical emission line diagnostics (Phillips et al. 1984), but is shown to be a Seyfert 1 by its double-peaked H $\alpha$ profile (Storchi-Bergmann et al. 1993). UV spectroscopy has revealed a few Myr old burst of star-formation in the central $9 \mathrm{pc}$ of the galaxy (Storchi-Bergmann et al. 2005). With the high spatial resolution of Herschel PACS, we can resolve the starburst ring and inner $600 \mathrm{pc}$ of NGC 1097 for the first time at wavelengths near the peak of the dust spectral energy distribution (SED).

\section{Observations and data reduction}

The galaxy NGC 1097 was observed with the PACS instrument (Poglitsch et al. 2010) on the Herschel Space Observatory (Pilbratt et al. 2010) on 2009 December 20 during the SDP. We obtained $15^{\prime}$ long scan-maps in two orthogonal directions at the medium scan speed $\left(20^{\prime \prime} \mathrm{s}^{-1}\right)$. The scan position angles $\left(45^{\circ} \mathrm{rel}-\right.$ ative to the scan direction) provide homogeneous coverage over the mapped region. The total on-source times per pixel were approximately 150,150 , and $300 \mathrm{~s}$ for 70,100 and $160 \mu \mathrm{m}$, respectively.

The raw data were reduced with HIPE (Ott 2010), version 3.0, build 455. Besides the standard steps leading to level-1 calibrated data, second-level deglitching and correction for offsets in the detector sub-matrices were performed. The data were then highpass-filtered using a median window of $5^{\prime}$ to remove the effects of bolometer sensitivity drifts and $1 / f$ noise. We masked out emission structures (visible in a first iteration of the map-making) with a $5^{\prime}$-wide mask before computing this running median to prevent subtraction of source emission. Although the filtering may remove some extended flux from the galaxy, because we are primarily interested in the very bright central $1^{\prime}$ of NGC 1097 this effect will be negligible. Finally, the data were projected onto a coordinate grid with $1^{\prime \prime}$ pixels.

After pipeline processing we applied flux correction factors from the PACS team to adjust the calibration. The current calibration has uncertainties of $\sim 10,10$, and $20 \%$ for the 70 , 100 and $160 \mu \mathrm{m}$ bands, respectively (Poglitsch et al. 2010). Because we aim to compare our PACS observations with ancillary data at other wavelengths, we adjusted the relative astrometry of the PACS observations to match that of the Spitzer $24 \mu \mathrm{m}$ from SINGS (Spitzer Infrared Nearby Galaxies Survey: Kennicutt et al. 2003). This was done by measuring the positions of background point-sources in the MIPS $24 \mu \mathrm{m}$ (MultiBand Imaging Photometer) and PACS $100 \mu \mathrm{m}$ images, adjusting the PACS $100 \mu \mathrm{m}$ astrometry, assuming the relative astrometry for the PACS bands is well-calibrated and transferring the solution to the other bands. The offset between the PACS and MIPS astrometry was $\sim 2^{\prime \prime}$. The one-sigma surface brightness sensitivities per pixel of the final maps are 5.9, 6.2 and 3.3 $\mathrm{MJy} \mathrm{sr}^{-1}$. In
Fig. 1 we show the three PACS images with a logarithmic stretch to highlight the spiral arms. Note that below we extract photometry from the images at their native resolution using apertures larger than the beam size of the lowest resolution map.

\section{Herschel PACS observations of the circumnuclear ring in NGC 1097}

The most prominent far-IR structure in NGC 1097 is its circumnuclear starburst ring, shown in Fig. 2 at a variety of wavelengths. The PACS angular resolution allows us to clearly separate the contribution of the ring and nucleus from the galaxy's emission for the first time at wavelengths that probe the peak of the dust SED. Summing the emission within a radius of $20^{\prime \prime}(1.8 \mathrm{kpc})$ of the center and comparing it with the total flux from the galaxy, we find that the ring and nucleus emit 75, 60 and 55\% of the total flux of NGC 1097 at 70, 100 and $160 \mu \mathrm{m}$, respectively (there is some galactic emission within $1.8 \mathrm{kpc}$ that is not associated with the ring or nucleus, but this component is negligible). These measurements imply that the SED of the more extended galactic emission peaks at longer wavelengths than the SED of the ring. Indeed, by fitting a modified blackbody to the MIPS and SPIRE photometry of the galaxy, Engelbracht et al. (2010) find that the central region of NGC 1097 is 22\% warmer than the disk.

The mid- and far-IR images of the ring in Fig. 2 show similar structures. The ring is continuous (i.e. no obvious gaps) with a series of bright knots. The same knots are visible in each PACS image, although at $160 \mu \mathrm{m}$ they are not well-resolved. At 70 and $100 \mu \mathrm{m}$, the surface brightness of the ring varies by less than $\pm 15 \%$ about the mean on $600 \mathrm{pc}$ scales. The variations at $24 \mu \mathrm{m}$ on the same spatial scales are $\pm 25 \%$. The similarities from midto far-IR suggest that dust temperatures are not varying substantially in the ring, which we quantify below.

It is interesting to note that the same pattern of bright knots is not observed in carbon monoxide (CO) (shown in panel $\mathrm{h}$ of Fig. 2) or other dense gas tracers at comparable resolution (Kohno et al. 2003; Hsieh et al. 2008). Instead the CO intensity peaks near the contact points and is much fainter over the rest of the ring. The differences between the far-IR and CO emission may be due to different $\mathrm{CO}$ excitation mechanisms in the shocked gas near the contact points or by the consumption and/or dissociation of molecular gas by star-formation events shortly downstream from the contact points. Three of the bright knots are also prominent in $3.5 \mathrm{~cm}$ radio continuum (as shown in panel f of Fig. 2). Beck et al. (2005) showed that the radio knots have a flatter radio spectral index than the rest of the ring, most likely due to either a contribution from free-free emission from HII regions or synchrotron emission from young supernova remnants (SNRs), which has an intrinsically flatter spectrum. Because young SNRs will heat only a small fraction of the dust, an enhancement of thermal radio continuum and dust heating in and around H II regions may be the best explanation for the origin of the coincident bright radio and far-IR knots.

In Fig. 3 we show the mid- and far-IR band ratios as a function of azimuthal angle. The surface brightness was measured in 9 azimuthal bins with inner and outer radii of 5 and $15^{\prime \prime}$ to adequately sample the PSF out to $100 \mu \mathrm{m}$. The largest variations are in the $24 / 70$ ratio, which peaks shortly downstream from the northernmost contact point, and varies by $\pm 15 \%$. The $70 / 100$ ratio varies by less than $\pm 5 \%$ around the ring. If there is a well-defined age gradient along the ring as predicted by the "pearls on a string" model, one might expect a gradient in 

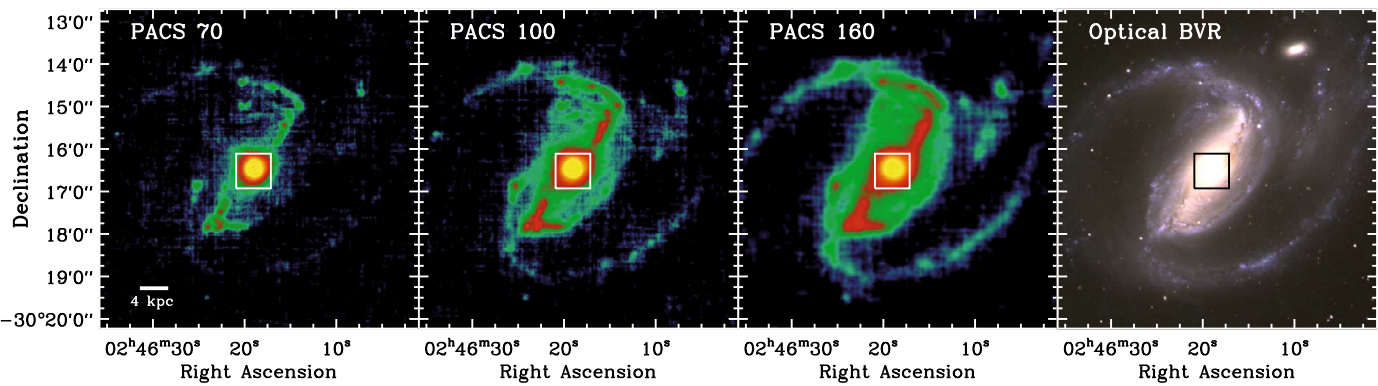

Fig. 1. PACS observations of NGC 1097 and an optical BVR image from the SINGS ancillary observations (Kennicutt et al. 2003) for comparison. The 70, 100 and $160 \mu \mathrm{m}$ bands are shown with a logarithmic stretch that highlights the spiral arms. The white square in each panel shows the location of the region presented in Fig. 2.
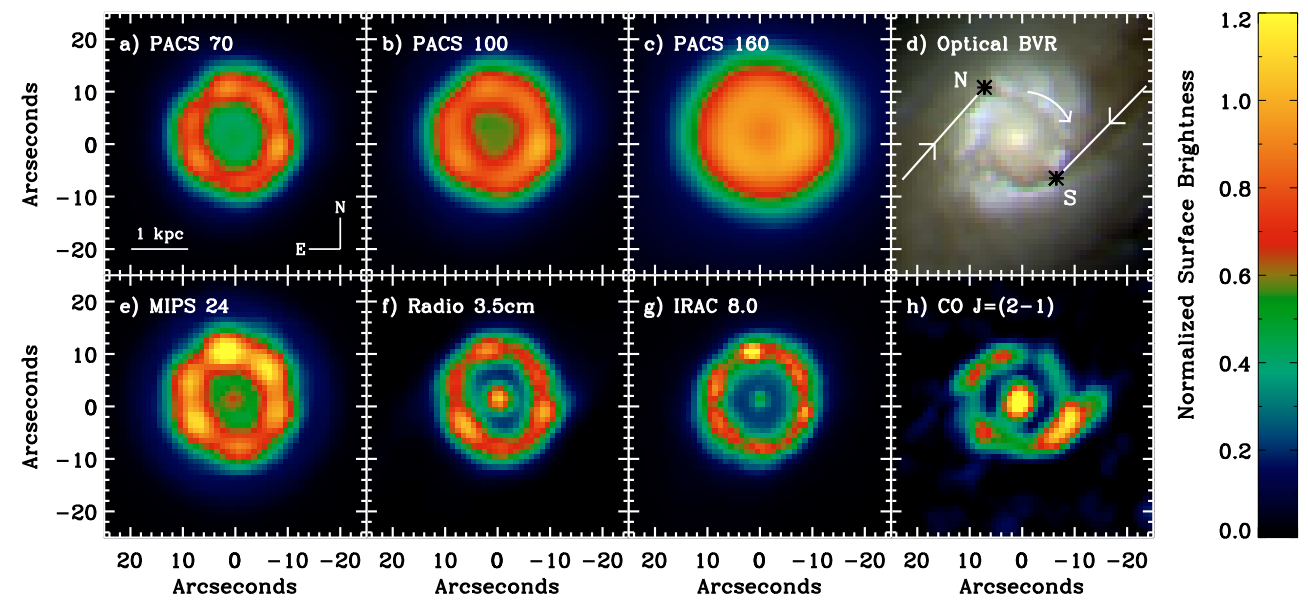

Fig. 2. Circumnuclear ring of NGC 1097 at a variety of wavelengths. Each image is shown at its native resolution: $\sim 6,7$ and $12^{\prime \prime}$ for PACS 70,100 and 160; $\sim 6^{\prime \prime}$ for MIPS 24; 4" for the $3.5 \mathrm{~cm}$ radio continuum (Beck et al. 2005) and CO $J=(2-1)$ (Hsieh et al. 2008); 2" for IRAC $8.0 \mu \mathrm{m}$; and $\sim 1^{\prime \prime}$ for the optical $B, V$, and $R$ imaging (Kennicutt et al. 2003). All images, except the three-color BVR image, are normalized to the surface brightness of the ring at the location $\left(-8^{\prime \prime},-1^{\prime \prime}\right)$, i.e. the brightest knot in the PACS 70 image. The approximate locations of the dust lanes that feed the circumnuclear ring are overlayed on the $B V R$ three-color image and the locations of the contact points are identified with black asterisks and labeled with "N" and "S". Material in the ring rotates clockwise as shown with the arrow in panel $\mathbf{h}$ ). Note that at this stretch the central source is not visible at $70 \mu \mathrm{m}$.

dust temperatures moving away from the contact points. In the youngest star-forming regions, the radiation field will be more intense due to the presence of the most massive and short-lived stars and the regions will be more compact. Both of these effects lead to hotter dust temperatures. For instance, Groves et al. (2008) modeled the spectra of the H II regions plus surrounding photo-dissociation region for star clusters with ages between 0.1-10 Myr. They find that for a cluster mass of $\sim 10^{5} M_{\odot}$ and an ISM pressure of $P / k \sim 10^{6} \mathrm{~K} \mathrm{~cm}^{-3}$ (approximately what has been deduced for the ISM in the circumnuclear ring by Hsieh et al. 2008), the 24/70 and 70/100 band ratios decrease by 90 and $70 \%$ (factors of $\sim 7$ and 3, respectively) as the cluster ages from 1 to 10 Myr. The Groves et al. (2008) models represent an upper bound to the band ratio variation we could expect if the ring was comprised solely of a well-defined sequence of aging clusters between 1-10 Myr old.

That we do not see large mid- and far-IR band ratio gradients does not rule out the existence of "pearls on a string" in favor of "popcorn" in NGC 1097, however. It may be the case that dust in the ring is predominantly heated by the radiation field from older stars (e.g. B stars with lifetimes of 10-100 Myr), which are uniformly distributed around the ring after a number of revolutions. Stellar population studies of the central $\mathrm{kpc}$ of NGC 1097 have shown that intermediate age stars make a considerable contribution to the UV radiation field in the vicinity of the ring (e.g. Bonatto et al. 1998). In this situation, the variation due to an age gradient would be diluted depending on the relative contribution of young clusters to the total dust heating. In addition, one might expect that given the fast dynamical time in the ring that local enhancements of dust heating are quickly wiped out. Stars and interstellar matter in NGC 1097's ring traverses the distance between the two contact points in $\sim 9$ Myr. Even assuming that the cluster formation happens instantaneously after entering the ring, there will still be an abundance of massive stars by the time the cluster crosses half the ring.

\section{Limits on the nuclear flux in the far-IR}

High resolution imaging at mid- and near-IR wavelengths of the nucleus of NGC 1097 shows an unresolved central point source (Prieto et al. 2005; Mason et al. 2007) which contains a nuclear starburst (Storchi-Bergmann et al. 2005) and the AGN. No observations can yet resolve the AGN or central starburst, but it is still possible to distinguish the contributions of the different sources to some degree. Mason et al. (2007), for instance, found that the 12 and $18 \mu \mathrm{m}$ emission arises primarily from dust heated by the nuclear starburst rather than the AGN torus. In NGC 1097 , previous far-IR flux limits for the nucleus were dominated by the starburst ring and provide limited information about the nuclear starburst or the AGN. With our Herschel observations, we can place limits on the flux arising in the central $\sim 600 \mathrm{pc}$. We use the PACS PSF observations of Vesta with a $20^{\prime \prime} \mathrm{s}^{-1}$ scan speed scaled to match the peak intensity of the point source we see in the center. Our scaled-PSF photometry is possible at 70 and 


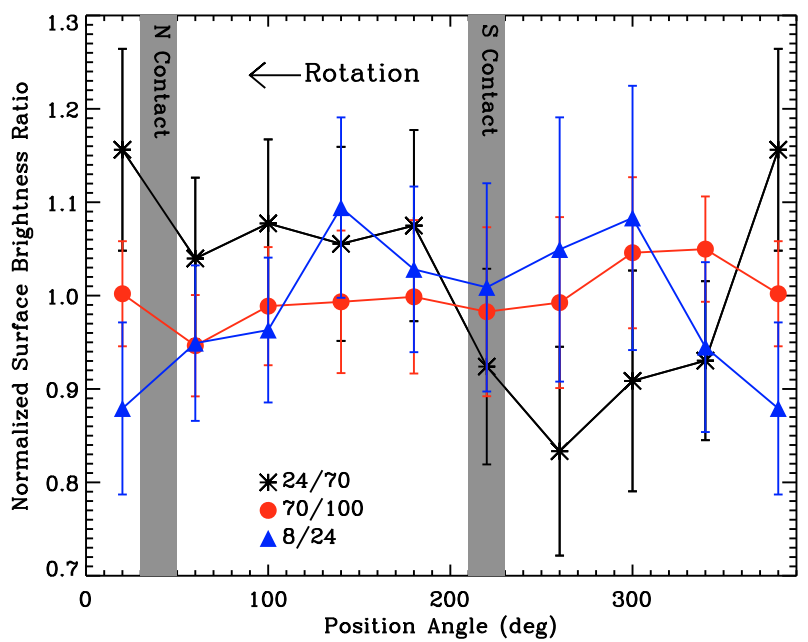

Fig. 3. Azimuthal variations of the mid- and far-IR band ratios with azimuth in the ring. The $x$-axis shows the position angle from North through East (increasing counter-clockwise from North in Fig. 2). Note that the first point is repeated at $380^{\circ}$. The band ratios are normalized to their mean values: $0.487,0.069$, and 0.776 for the $8.0 / 24,24 / 70$ and $70 / 100$ ratios, respectively. The contact points of the dust lanes and the direction of rotation for the ring are labeled on the plot.

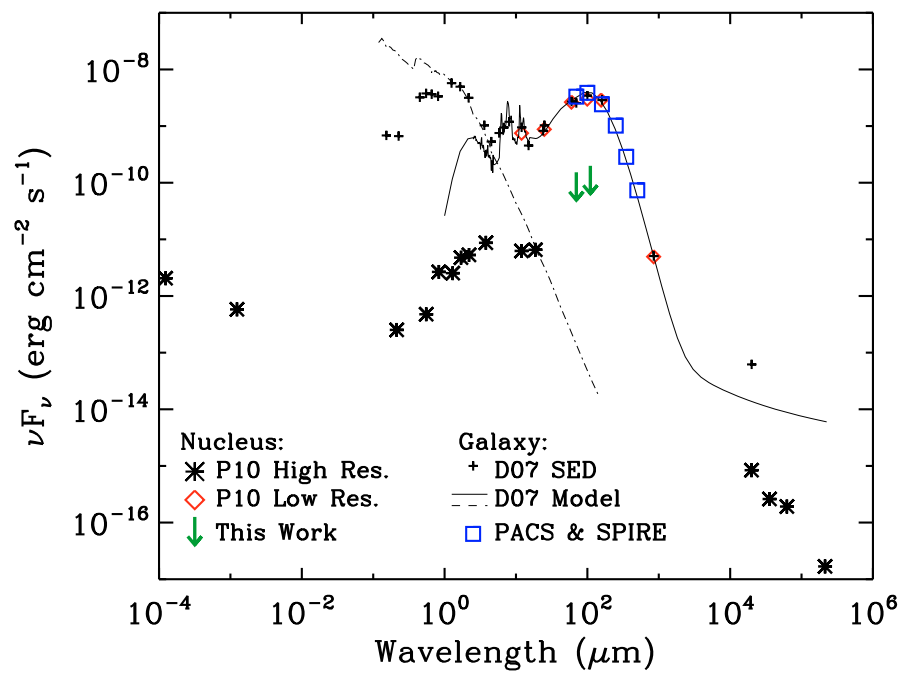

Fig. 4. SED of the nuclear region from the compilation of Prieto et al. (2010, P10) and the SED of the whole galaxy from Dale et al. (2007, D07). The black crosses show the D07 SED overlaid with their bestfit model to the MIPS observations. The blue squares show PACS and SPIRE fluxes for NGC 1097 from KINGFISH observations (see Engelbracht et al. 2010, for discussion of the SPIRE observations). The black asterisks show observations at high spatial resolution (on the order of $1^{\prime \prime}$ or less). The red points are lower resolution IR measurements of the central region which are dominated by the flux from the starburst ring. The green arrows show the new limits we can place on the nuclear flux using our PACS observations.

$100 \mu \mathrm{m}$, but not at $160 \mu \mathrm{m}$ where the central source is not wellresolved. The best scaled PSF has a total flux of 3.5 and $7.3 \mathrm{Jy}$ at 70 and $100 \mu \mathrm{m}$. Without more detailed modeling of the nuclear region these measurements should only be considered upper limits. However, they improve constraints on the nuclear flux by more than an order of magnitude as shown in Fig. 4, which presents the SED of the nucleus from a compilation by Prieto et al. (2010).

\section{Conclusions}

We have presented Herschel PACS observations from KINGFISH of the inner $\mathrm{kpc}$ of the barred spiral galaxy NGC 1097. These are the first observations to resolve a starburst ring at wavelengths probing the peak of the dust SED. We show a comparison of the ring in a variety of tracers and find similar bright knots in the mid- and far-IR and radio continuum. These knots do not correspond to the same knots traced by CO. We find modest variation azimuthally in the mid- and far-IR band ratios suggesting that either there is no azimuthal age gradient, as would be predicted by the "pearls on a string" mode of star-formation, that dust heating is dominated by an older stellar population and/or that the dust heating variations get quickly erased over the short ring orbital period ( $\sim 18$ Myr). Finally, we place an order-of-magnitude tighter constraint on the far-IR emission originating in the central $\sim 600 \mathrm{pc}$ of the galaxy.

Acknowledgements. The authors thank R. Beck for the radio continuum data and P.-Y. Hsieh for the $\mathrm{CO}$ data. K.S. would like to thank G. van de Ven and L. Burtscher for useful discussions. PACS has been developed by a consortium of institutes led by MPE (Germany) and including UVIE (Austria); KU Leuven, CSL, IMEC (Belgium); CEA, LAM (France); MPIA (Germany); INAF- IFSI/OAA/OAP/OAT, LENS, SISSA (Italy); IAC (Spain). This development has been supported by the funding agencies BMVIT (Austria), ESAPRODEX (Belgium), CEA/CNES (France), DLR (Germany), ASI/INAF (Italy), and CICYT/MCYT (Spain).

\section{References}

Athanassoula, E. 1992, MNRAS, 259, 345

Beck, R., Fletcher, A., Shukurov, A., et al. 2005, A\&A, 444, 739 Beirão, P., et al. 2010, A\&A, 518, L60

Böker, T., Falcón-Barroso, J., Schinnerer, E., Knapen, J. H., \& Ryder, S. 2008, AJ, 135, 479

Bonatto, C., Pastoriza, M. G., Alloin, D., \& Bica, E. 1998, A\&A, 334, 439

Combes, F. 1996, in Barred Galaxies, ed. R. Buta, D. A. Crocker, \& B. G. Elmegreen, IAU Coll., 157, ASP Conf. Ser., 91, 286

Combes, F., \& Gerin, M. 1985, A\&A, 150, 327

Dale, D. A., Gil de Paz, A., Gordon, K. D., et al. 2007, ApJ, 655, 863 Elmegreen, B. G. 1994, ApJ, 425, L73

Engelbracht, C. W., et al. 2010, A\&A, 518, L56

Groves, B., Dopita, M. A., Sutherland, R. S., et al. 2008, ApJS, 176, 438

Hsieh, P., Matsushita, S., Lim, J., Kohno, K., \& Sawada-Satoh, S. 2008, ApJ, 683,70

Hummel, E., van der Hulst, J. M., \& Keel, W. C. 1987, A\&A, 172, 32 Kennicutt, Jr., R. C., Armus, L., Bendo, G., et al. 2003, PASP, 115, 928

Kohno, K., Ishizuki, S., Matsushita, S., Vila-Vilaró, B., \& Kawabe, R. 2003, PASJ, 55, L1

Kormendy, J., \& Kennicutt, Jr., R. C. 2004, ARA\&A, 42, 603

Maoz, D., Barth, A. J., Ho, L. C., Sternberg, A., \& Filippenko, A. V. 2001, AJ, 121,3048

Maoz, D., Barth, A. J., Sternberg, A., et al. 1996, AJ, 111, 2248

Mason, R. E., Levenson, N. A., Packham, C., et al. 2007, ApJ, 659, 241

Mazzuca, L. M., Knapen, J. H., Veilleux, S., \& Regan, M. W. 2008, ApJS, 174, 337

Norman, C. A., Sellwood, J. A., \& Hasan, H. 1996, ApJ, 462, 114

Ott, S. 2010, in Astronomical Data Analysis Software and Systems XIX, ed. Y. Mizumoto, K.-I. Morita, \& M. Ohishi, ASP Conf. Ser., in press Phillips, M. M., Pagel, B. E. J., Edmunds, M. G., \& Diaz, A. 1984, MNRAS, 210, 701

Pilbratt, G. L., et al. 2010, A\&A, 518, L1

Poglitsch, A., et al. 2010, A\&A, 518, L2

Prieto, M. A., Maciejewski, W., \& Reunanen, J. 2005, AJ, 130, 1472

Prieto, M. A., Reunanen, J., Tristram, K. R. W., et al. 2010, MNRAS, 402, 724

Storchi-Bergmann, T., Baldwin, J. A., \& Wilson, A. S. 1993, ApJ, 410, L11

Storchi-Bergmann, T., Wilson, A. S., \& Baldwin, J. A. 1996, ApJ, 460, 252

Storchi-Bergmann, T., Nemmen, R. S., Spinelli, P. F., et al. 2005, ApJ, 624, L13

van de Ven, G., \& Chang, P. 2009, ApJ, 697, 619

Willick, J. A., Courteau, S., Faber, S. M., et al. 1997, ApJS, 109, 333

Page 5 is available in the electronic edition of the journal at http://www . aanda.org 
1 Max-Planck-Institut für Astronomie, Königstuhl 17, 69117 Heidelberg, Germany

e-mail: sandstrom@mia.de

2 CEA/DSM/DAPNIA/Service d'Astrophysique, UMR AIM, CE Saclay, 91191 Gif sur Yvette Cedex, France

3 Institute of Astronomy, University of Cambridge, Madingley Road, Cambridge CB3 0HA, UK

4 Department of Astronomy, University of Massachusetts, Amherst, MA 01003, USA

5 NASA Herschel Science Center, IPAC, California Institute of Technology, Pasadena, CA 91125, USA

${ }^{6}$ Spitzer Science Center, California Institute of Technology, MC 314-6, Pasadena, CA 91125, USA

7 Department of Astronomy, University of Maryland, College Park, MD 20742, USA

8 Leiden Observatory, Leiden University, PO Box 9513, 2300 RA Leiden, The Netherlands

9 Department of Physics and Astronomy, University of Toledo, Toledo, OH 43606, USA

10 Department of Physics \& Astronomy, University of Wyoming, Laramie, WY 82071, USA

11 Department of Astrophysical Sciences, Princeton University, Princeton, NJ 08544, USA
12 Steward Observatory, University of Arizona, Tucson, AZ 85721, USA

13 Departamento de Astrofisica, Facultad de Ciencias Fisicas, Universidad Complutense Madrid, Ciudad Universitaria, Madrid, 28040, Spain

14 Space Telescope Science Institute, 3700 San Martin Drive, Baltimore, MD 21218, USA

15 Tianjin Astrophysics Center, Tianjin Normal University, Tianjin 300387, China

16 INAF - Osservatorio Astrofisico di Arcetri, Largo E. Fermi 5, 50125 Firenze, Italy

17 Department of Physics and Astronomy, SUNY Stony Brook, Stony Brook, NY 11794-3800, USA

18 Hubble Fellow, National Radio Astronomy Observatory, 520 Edgemont Road, Charlottesville, VA 22903, USA

19 Institut d'Astrophysique de Paris, UMR7095 CNRS, Université Pierre \& Marie Curie, 98 bis Boulevard Arago, 75014 Paris, France

20 ICRAR, M468, University of Western Australia, 35 Stirling Hwy, Crawley, WA 6009, Australia

21 Department of Physics \& Astronomy, McMaster University, Hamilton, Ontario L8S 4M1, Canada 